\title{
The exponentiated generalized extended exponential distribution
}

\author{
Thiago A. N. de Andrade $^{1 *}$, Marcelo Bourguignon ${ }^{2 \dagger}$, Gauss M. Cordeiro ${ }^{3 *}$ \\ ${ }^{1,3}$ Departamento de Estat'istica, Universidade Federal de Pernambuco \\ ${ }^{2}$ Departamento de Estat' istica, Universidade Federal do Rio Grande do Norte
}

\begin{abstract}
We introduce and study a new four-parameter lifetime model named the exponentiated generalized extended exponential distribution. The proposed model has the advantage of including as special cases the exponential and exponentiated exponential distributions, among others, and its hazard function can take the classic shapes: bathtub, inverted bathtub, increasing, decreasing and constant, among others. We derive some mathematical properties of the new model such as a representation for the density function as a double mixture of Erlang densities, explicit expressions for the quantile function, ordinary and incomplete moments, mean deviations, Bonferroni and Lorenz curves, generating function, R'enyi entropy, density of order statistics and reliability. We use the maximum likelihood method to estimate the model parameters. Two applications to real data illustrate the flexibility of the proposed model.
\end{abstract}

Key words: Erlang distribution, Extended exponential distribution, Hazard rate function, Lifetime distribution, Moments.

\section{Introduction}

The exponential distribution is a very popular statistical model and, probably, is one of the parametric models most extensively applied in several fields (Lemonte, 2013). The popularity of this distribution can be explained, perhaps, by the simplicity of their cumulative function, which involves only one unknown parameter $\lambda>0$ and takes a simple form $G(x)=1-\mathrm{e}^{-\lambda x}$, for $x>0$, in addition to having constant hazard rate function (hrf). Due to its importance, several studies introducing and/or studying extensions of the exponential distribution are available in the literature. Here, we refer to the following papers: Gupta et al. (1998), Gupta and Kundu (2001), Nadarajah and Haghighi (2011) and Lemonte (2013), to mention a few. Recently, Gómez et al. (2014) introduced the extended exponential ( $\mathcal{E} \mathcal{E}$ for short) distribution, whose cumulative distribution function (cdf) and probability density function (pdf) (for $\mathrm{x}>0$ ) are given by

$$
G(x ; \alpha, \beta)=\frac{\alpha+\beta-(\beta+\alpha+\alpha \beta x) \mathrm{e}^{-\alpha x}}{\alpha+\beta}
$$


and

$$
g(x ; \alpha, \beta)=\frac{\alpha^{2}(1+\beta x) \mathrm{e}^{-\alpha x}}{\alpha+\beta},
$$

respectively, where $\alpha>0$ and $\beta \geq 0$.

We emphasize that the density (2) can be also obtained as a special case of the generalized Lindley distribution proposed by Zakerzadeh and Dolati (2009). However, these latter authors do not address this particular case in their research.

Several mathematical properties of the $\mathcal{E} \mathcal{E}$ distribution, including expectation, variance, moment generating function (mgf), asymmetry and kurtosis coefficients, among others, were studied by Gómez et al. (2014). In particular, they proved that the density of the $\mathcal{E} \mathcal{E}$ model is a mixture of the exponential and gamma densities. We believe that the addition of parameters to the $\mathcal{E} \mathcal{E}$ model may generate new distributions with great adjustment capability and, for this reason, we propose a generalization of it.

In a recent paper, Cordeiro et al. (2013) proposed a new way of adding two parameters to a continuous distribution. For a given continuous baseline cdf $G(x)$, and $x \in R$, they defined the exponentiated generalized $(\boldsymbol{E} \boldsymbol{g})$ class of distributions with two extra shape parameters a $>0$ and b $>0$ and $\operatorname{cdf} F(x)$ and $\operatorname{pdf} f(x)$ given by

$$
F(x)=\left\{1-[1-G(x)]^{a}\right\}^{b}
$$

and

$$
f(x)=a b[1-G(x)]^{a-1}\left\{1-[1-G(x)]^{a}\right\}^{b-1} g(x),
$$

respectively, in which are implicit the dependence on the parameters of $G(x)$.

To illustrate the flexibility of the $\boldsymbol{E g}$ model, Cordeiro et al. (2013) applied (3) to extend some well-known distributions such as the Fréchet, normal, gamma and Gumbel distributions. Moreover, they presented several properties for the $\boldsymbol{\varepsilon} \boldsymbol{g}$ class, which provide motivations to adopt this generator. Next, we discuss some of these motivations. The first important point to note is the simplicity of equations (3) and (4). They have no complicated functions and will be always tractable when the cdf and pdf of the baseline distribution have simple analytic expressions. It is very easy, for example, to obtain the inverse of the cdf (3). Another important feature is that the $\boldsymbol{E} \boldsymbol{g}$ model contains as especial cases the two classes of Lehmann's alternatives. In fact, for $a=1$, (3) reduces to $F(x)=G(x)^{b}$ and, for $b=1$, we obtain $F(x)=1-[1-G(x)]^{a}$, which correspond to the cdf's of the Lehmann type I and II families (Lehmann, 1953), respectively. For this reason, the $\boldsymbol{E} \boldsymbol{g}$ model encompasses both Lehmann type I and type II classes. So, the $\boldsymbol{E} \boldsymbol{g}$ family can be derived from a double transformation using these classes. The two extra parameters $a$ and $b$ in the density (4) can control both tail weights, allowing generate flexible distributions, with heavier or lighter tails, as appropriate. There is also an attractive physical interpretation of the model (3) when $a$ and $b$ are positive integers. This interpretation is described in Cordeiro and Lemonte (2014).

The above properties and many others have been discussed and explored in recent works for the $\boldsymbol{E} \boldsymbol{g}$ class. Here, we refer to the papers: Cordeiro et al. (2014), Cordeiro and Lemonte (2014), Elbatal and Muhammed (2014), Oguntunde et al. (2014) and da Silva et al. (2015), which used 
the $\boldsymbol{\varepsilon} \boldsymbol{g}$ class to extend the Burr III, Birnbaum-Saunders, inverse Weibull, inverted exponential and generalized gamma distributions, respectively.

In this paper, we define the exponentiated generalized extended exponential $(\boldsymbol{E} \boldsymbol{g} \boldsymbol{E} \boldsymbol{E})$ distribution by inserting (1) in equation (3). The EGEE model includes as special cases the exponential, Lindley and exponentiated exponential distributions, among others, which are very important statistical models, specially for applied works. The new density function is a double linear mixture of Erlang densities, and thus several properties of the $\mathcal{E} g \mathcal{E} \mathcal{E}$ model can be simplified from this relationship. Moreover, the proposed model has monotonic and non-monotonic hrf's. We hope that the new distribution can be widely used for data modeling in areas such as economics, finance, reliability, biology and medicine, among others.

The rest of the paper is organized as follows. In Section 2, we give the density and hazard functions of the $\boldsymbol{E} \boldsymbol{g} \mathcal{E} \boldsymbol{E}$ model and corresponding plots for selected parameter values. We provide expressions for the cumulative and reversed hazard functions. In Section 3, we investigate the shapes of the $\boldsymbol{E} \boldsymbol{g} \mathcal{E} \mathcal{E}$ density function. In Section 4, we derive several mathematical properties of the proposed model, including mixture representations for the density and cumulative functions, explicit expressions for the quantile and generating functions, ordinary and incomplete moments, among others. Estimation and inference by maximum likelihood are discussed in Section 5. Two applied results are presented in Section 6. Section 7 provides concluding remarks.

\section{The $\mathcal{E} g \mathcal{E} \mathcal{E}$ distribution}

The cdf and pdf of the $\mathcal{E} \boldsymbol{g} \mathcal{E} \mathcal{E}$ distribution (by omitting the dependence on the parameters a > $0, b>0, \alpha>0$ and $\beta \geq 0$ ), for $x>0$, are given by

$$
F(x)=\frac{\left[(\alpha+\beta)^{a}-(\beta+\alpha+\alpha \beta x)^{a} \mathrm{e}^{-a \alpha x}\right]^{b}}{(\alpha+\beta)^{a b}}
$$

and

$$
f(x)=\frac{a b \alpha^{2}}{(\alpha+\beta)^{a b}}(1+\beta x) \mathrm{e}^{-a \alpha x}(\beta+\alpha+\alpha \beta x)^{a-1}\left[(\alpha+\beta)^{a}-(\beta+\alpha+\alpha \beta x)^{a} \mathrm{e}^{-a \alpha x}\right]^{b-1},
$$

respectively. Henceforth, a continuous random variable having pdf (6) is denoted by $\mathrm{X} \sim \mathcal{E} \boldsymbol{g} \mathcal{E} \mathcal{E}$ $(a, b, \alpha, \beta)$.

Several distributions are special cases of the $\mathcal{E} \boldsymbol{g} \mathcal{E} \mathcal{E}$ model. Here, we mention some of them. Clearly, the $\mathcal{E} \mathcal{E}$ distribution is a basic exemplar when $a=b=1$. The exponential and Lindley distributions are obtained from (6) by setting $\beta=0$ and $\beta=1$, respectively, in addition to $a=b$ $=1$. The exponentiated generalized exponential $(\boldsymbol{E} \boldsymbol{g} \mathcal{E})$ model comes from (6) by setting $\beta=0$ and the exponentiated generalized Lindley $(\boldsymbol{E} \boldsymbol{g} \boldsymbol{L})$ distribution follows when $\beta=1$. The $\boldsymbol{E} \boldsymbol{g} \mathcal{E} \mathcal{E}$ model also includes the Lehmann type I and type II transformations of the $\mathcal{E} \mathcal{E}$, exponential and Lindley distributions. For example, the well-known exponentiated exponential distribution (Gupta et al., 1998), also referred in the literature as the generalized exponential distribution, follows when $\beta$

$=0$ and $a=1$. For a brief discussion and some properties of the exponentiated exponential 
distribution, see a recent paper by Lemonte (2013). The exponentiated Lindley $(\boldsymbol{E} \boldsymbol{L})$ model by Nadarajah et al. (2011) (they called the generalized Lindley distribution, but here we adopt the EL terminology) comes when $a=\beta=1$.

The hrf and reversed hazard rate function (rhrf) of $\mathrm{X}$ are given by

$$
h(x)=\frac{a b \alpha^{2}(1+\beta x) \mathrm{e}^{-a \alpha x}(\beta+\alpha+\alpha \beta x)^{a-1}\left[(\alpha+\beta)^{a}-(\beta+\alpha+\alpha \beta x)^{a} \mathrm{e}^{-a \alpha x}\right]^{b-1}}{(\alpha+\beta)^{a b}-\left[(\alpha+\beta)^{a}-(\beta+\alpha+\alpha \beta x)^{a} \mathrm{e}^{-a \alpha x}\right]^{b}}
$$

and

$$
\tau(x)=\frac{a b \alpha^{2}(1+\beta x) \mathrm{e}^{-a \alpha x}(\beta+\alpha+\alpha \beta x)^{a-1}}{\left[(\alpha+\beta)^{a}-(\beta+\alpha+\alpha \beta x)^{a} \mathrm{e}^{-a \alpha x}\right]},
$$

respectively.

Plots of the $\mathcal{E} \boldsymbol{g} \mathcal{E} \mathcal{E}$ density for selected parameter values are displayed in Figure 1. Figure 2 provides some possible shapes of the $\mathcal{E} \boldsymbol{g} \mathcal{E} \mathcal{E}$ hazard function for appropriate parameter values, including bathtub, inverted bathtub, increasing, decreasing and constant shapes. These plots indicate that the $\mathcal{E} \boldsymbol{g} \mathcal{E} \mathcal{E}$ model is fairly flexible and can be used to fit several types of positive data 


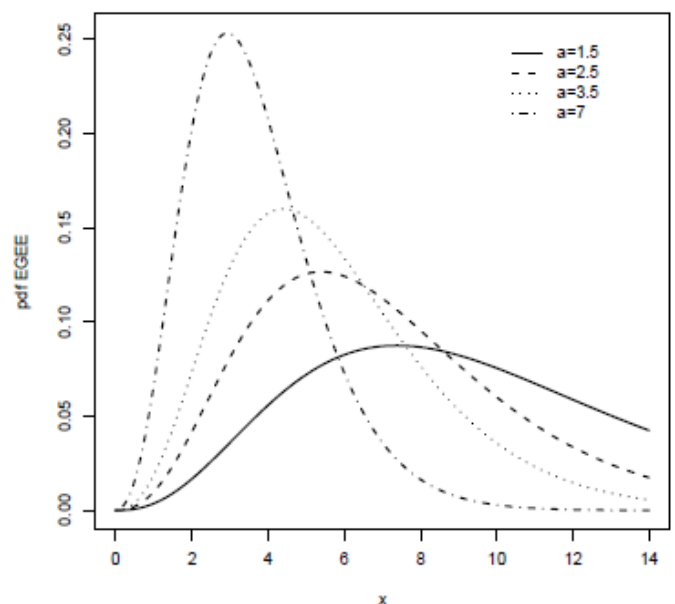

(a) $b=2, \alpha=0.2$ and $\beta=6$

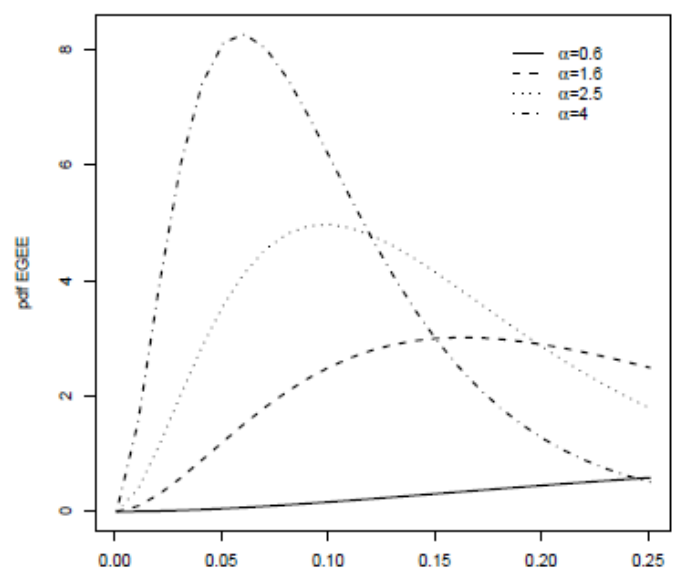

(c) $a=5, b=3$ and $\beta=0.3$

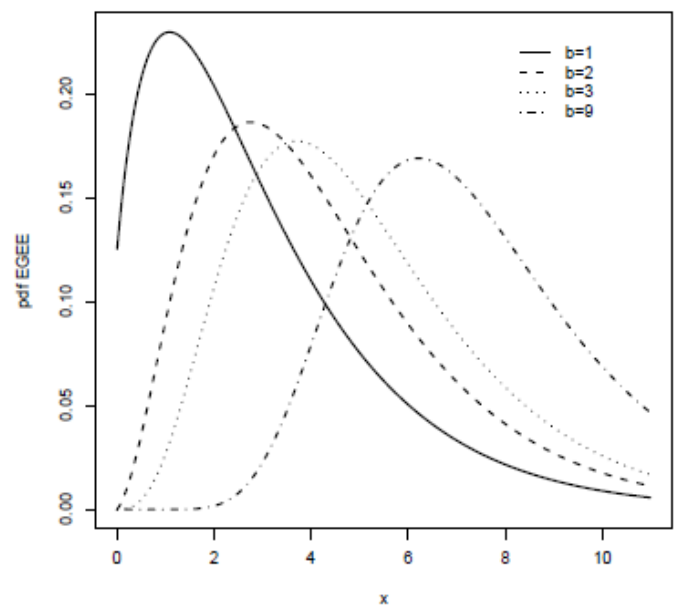

(b) $a=0.5, \alpha=1$ and $\beta=3$

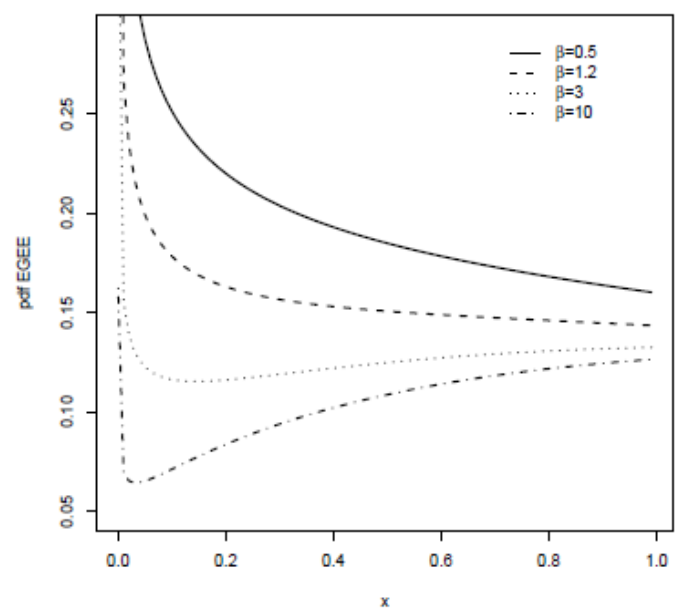

(d) $a=0.4, b=0.8$ and $\alpha=0.6$

Figure 1: Plots of the $\mathcal{E} \boldsymbol{g} \mathcal{E} \mathcal{E}$ density function for some parameter values.

\section{Shapes}

The first derivative of $\log \{f(x)\}$ for the $\mathcal{E} \boldsymbol{g} \mathcal{E} \mathcal{E}$ model is given by

$$
\frac{\mathrm{d} \log \{f(x)\}}{\mathrm{d} x}=-a \alpha+\frac{\beta}{1+\beta x}+\frac{(a-1) \alpha \beta}{z(x)}+\frac{a(b-1) \alpha z(x)^{a}[1-\beta / z(x)] \mathrm{e}^{-a \alpha x}}{(\alpha+\beta)^{a}-z(x)^{a} \mathrm{e}^{-a \alpha x}},
$$




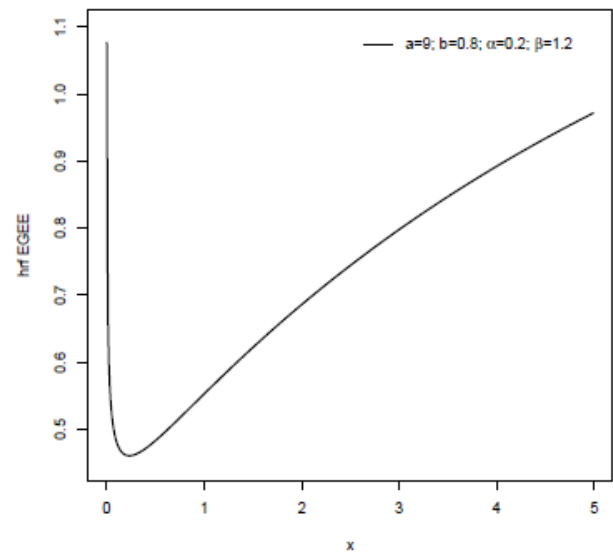

(a) bathtub

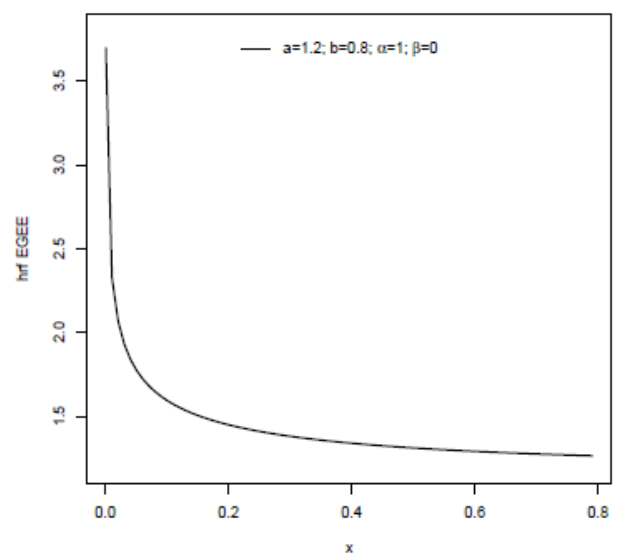

(c) decreasing

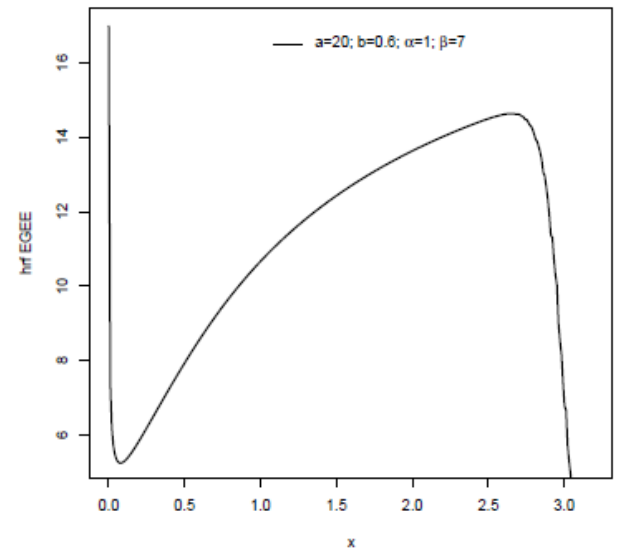

(e) decreasing-increasing-decreasing

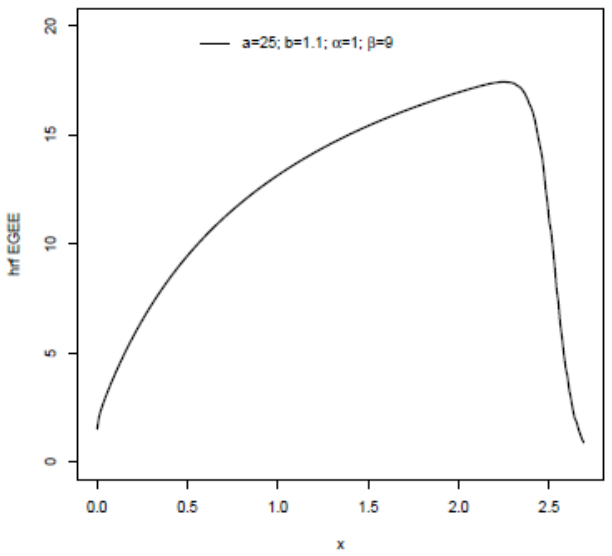

(b) inverted bathtub

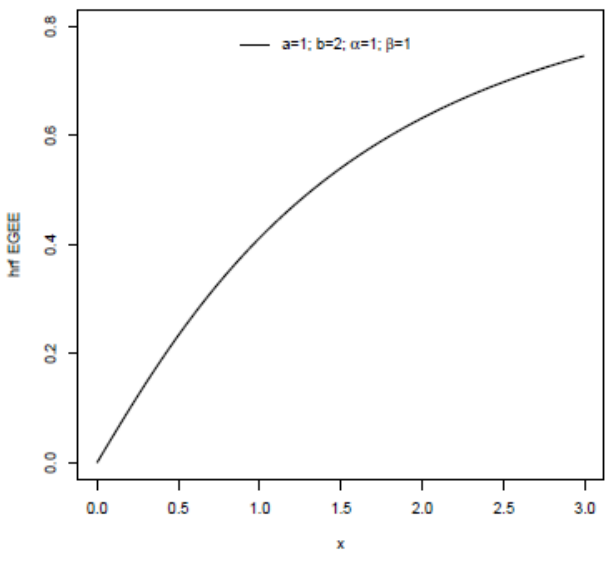

(d) increasing

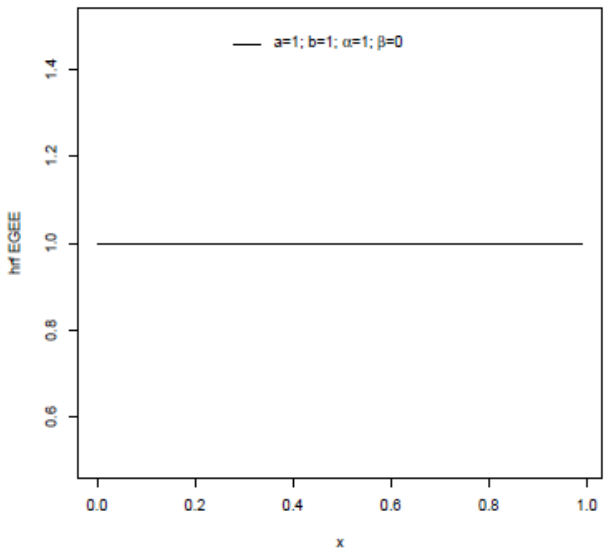

(f) constant

Figure 2: Plots of the $\mathcal{E} \boldsymbol{g} \mathcal{E} \mathcal{E}$ hazard function for some parameter values. 
where $z(x)=\beta+\alpha+\alpha \beta x$.

Thus, the critical values of $f(x)$ are the roots of the equation:

$$
\frac{a(b-1) \alpha z(x)^{a}[1-\beta / z(x)] \mathrm{e}^{-a \alpha x}}{(\alpha+\beta)^{a}-z(x)^{a} \mathrm{e}^{-a \alpha x}}=a \alpha-\frac{\beta}{1+\beta x}-\frac{(a-1) \alpha \beta}{z(x)} .
$$

If the point $x=x_{0}$ is a root of (7), then we can classify it as local maximum, local minimum or inflection point when $\lambda\left(x_{0}\right)<0, \lambda\left(x_{0}\right)>0$ and $\lambda\left(x_{0}\right)=0$, respectively, where $\lambda(x)=\mathrm{d}^{2} \log \{f(x)\} / \mathrm{d} x^{2}$ is given by

$$
\begin{aligned}
\lambda(x)= & -\frac{\beta^{2}}{(1+\beta x)^{2}}-\frac{(a-1) \alpha^{2} \beta^{2}}{z(x)^{2}}-\frac{(b-1)\left\{a \alpha z(x)^{a}[1-\beta / z(x)] \mathrm{e}^{-a \alpha x}\right\}^{2}}{\left[(\alpha+\beta)^{a}-z(x)^{a} \mathrm{e}^{-a \alpha x}\right]^{2}} \\
& +\frac{a(b-1) \alpha^{2} z(x)^{a}\left[a \beta z(x)^{-1}-a-(a-1) \beta^{2} z(x)^{-2}+a \beta z(x)^{-1}\right] \mathrm{e}^{-a \alpha x}}{(\alpha+\beta)^{a}-z(x)^{a} \mathrm{e}^{-a \alpha x}} .
\end{aligned}
$$

\section{Properties}

In this section, we study some structural properties of the $\mathcal{E} g \mathcal{E} \mathcal{E}$ distribution.

\subsection{A useful representation}

First, we derive simple representations for the density and cumulative functions of the $\boldsymbol{E} \boldsymbol{g} \mathcal{E} \mathcal{E}$ distribution. The starting point of our approach is the class of exponentiated distributions, which has been widely explored in recent works. A comprehensive review of these publications can be found in a recent paper by Tahir and Nadarajah (2015). For an arbitrary continuous baseline cdf $G(x)$, a random variable $Y$ is said to have the exponentiated- $\boldsymbol{g}$ ("exp- $\boldsymbol{g}$ " for short) distribution with power parameter $a>0$, say $Y \sim \exp -g(a)$, if its cdf and pdf are $H_{a}(x)=G(x)^{a}$ and $h_{a}(x)=a$ $g(x) G(x)^{a-1}$, respectively. Thus, "exp-g" denotes the Lehmann type I transformation of $G(x)$. Based on some results in Cordeiro and Lemonte (2014), we can express the $\boldsymbol{E} \boldsymbol{g}$ cdf (3) as

$$
F(x)=\sum_{j=0}^{\infty} w_{j+1} H_{j+1}(x),
$$

where $\quad w_{j+1}=\sum_{m=1}^{\infty}(-1)^{j+m+1}\left(\begin{array}{c}b \\ m\end{array}\right)\left(\begin{array}{c}m a \\ j+1\end{array}\right)$ and $H_{j+1}(x)=G(x)^{j+1}$ is the exp- $\boldsymbol{g}$ cdf with power parameter $j+1$. By differentiating (8), we obtain a similar mixture representation for $\mathrm{f}(\mathrm{x})$ as

$$
f(x)=\sum_{j=0}^{\infty} w_{j+1} h_{j+1}(x),
$$

where $h_{j+1}(x)=d H_{j+1}(x)=d x$. 
By using (8) and (9) for the $\mathcal{E} \mathcal{E}$ distribution (1), $h_{j+1}(x)$ becomes the exp- $\mathcal{E} \mathcal{E}$ pdf with power parameter $j+1$ (for $\mathrm{j} \geq 0$ ) given by

$$
h_{j+1}(x)=\frac{(j+1) \alpha^{2}}{(\alpha+\beta)^{j+1}}(1+\beta x) \mathrm{e}^{-\alpha x}\left[\alpha+\beta-(\beta+\alpha+\alpha \beta x) \mathrm{e}^{-\alpha x}\right]^{j} .
$$

Combining equations (9) and (10) we have an important result: the $\mathcal{E} \boldsymbol{g} \mathcal{E} \mathcal{E}$ density function is a linear mixture of exp- $\mathcal{E} \mathcal{E}$ densities. This result can be used to derive some mathematical properties of $X$.

Next, we apply the binomial expansion in equation (10) in order to obtain a simple

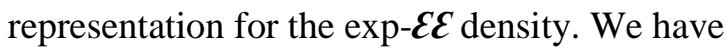

$$
h_{j+1}(x)=\sum_{k=0}^{j} \sum_{\ell=0}^{k} \sum_{i=0}^{\ell+1} \frac{(j+1)(-1)^{k} \alpha^{\ell+2} \beta^{i+k-\ell}}{(\alpha+\beta)^{k+1}}\left(\begin{array}{l}
j \\
k
\end{array}\right)\left(\begin{array}{c}
k \\
\ell
\end{array}\right)\left(\begin{array}{c}
\ell+1 \\
i
\end{array}\right) x^{i} \mathrm{e}^{-\alpha(k+1) x} .
$$

By interchanging $\sum_{\ell=0}^{k} \sum_{i=0}^{\ell+1}$ by $\sum_{i=0}^{k+1} \sum_{\ell=\delta_{i}}^{k}$ in the last equation, where

$$
\delta_{i}= \begin{cases}0, & \text { if } i=0,1 \\ i-1, & \text { if } i \geq 2\end{cases}
$$

and, after a simple algebraic manipulation, we obtain

$$
h_{j+1}(x)=\sum_{k=0}^{j} \sum_{i=0}^{k+1} p_{k, i}^{(j+1)} \pi(x ; i+1,(k+1) \alpha),
$$

where

$$
p_{k, i}^{(j+1)}=\sum_{\ell=\delta_{i}}^{k} \frac{(j+1)(-1)^{k} \alpha^{\ell-i+1} \beta^{i+k-\ell} i !}{(k+1)^{i+1}(\alpha+\beta)^{k+1}}\left(\begin{array}{l}
j \\
k
\end{array}\right)\left(\begin{array}{c}
k \\
\ell
\end{array}\right)\left(\begin{array}{c}
\ell+1 \\
i
\end{array}\right) .
$$

Here, $\pi(x ; i+1,(k+1) \alpha)$ denotes the pdf of the Erlang distribution with shape parameter $i+1$ (for $i \geq 0$ ) and scale parameter $(k+1) \alpha$. If $Z$ is the Erlang random variable with shape parameter $s$ $(=1,2,3, \ldots)$ and scale parameter $\lambda>0$, its pdf is given by $\pi(z ; s, \lambda)=\lambda^{s} z^{s-1} \mathrm{e}^{-\lambda z} /(s-1) !$.

Second, combining equations (9) and (11) and changing $\sum_{j=0}^{\infty} \sum_{k=0}^{j}$ by $\sum_{k=0}^{\infty} \sum_{j=k}^{\infty}$, the density function of the $\mathcal{E} \boldsymbol{g} \mathcal{E} \mathcal{E}$ model reduces to

$$
f(x)=\sum_{k=0}^{\infty} \sum_{i=0}^{k+1} v_{k, i} \pi(x ; i+1,(k+1) \alpha)
$$

where $v_{k, i}=\sum_{j=k}^{\infty} w_{j+1} p_{k, i}^{(j+1)}$

Equation (13) is the main result of this section. It gives the density function of $X$ as a double linear mixture of Erlang densities. This result is important to obtain some mathematical properties of $X$ such as the ordinary and incomplete moments, generating function and mean deviations from those of the Erlang distribution. We can take the upper limit of $k$ to be equal 20 in equation (13) for most practical purposes. 


\subsection{Quantile function}

For many applications it is important to determine the quantile function (qf) of $X$. Based on this function, we can, for example, generate variates and obtain the median of the $\boldsymbol{\varepsilon} \boldsymbol{g} \mathcal{E} \mathcal{E}$ distribution. By inverting (5), the qf of $X$ can be expressed as

$$
Q(u)=-\frac{1}{\alpha}-\frac{1}{\beta}-\frac{1}{\alpha} \mathrm{W}\left\{-\frac{1}{\beta}(\alpha+\beta)\left(1-u^{1 / b}\right)^{1 / a} \exp [-(\alpha+\beta) / \beta]\right\},
$$

where $0<\mathrm{u}<1$ and $\mathrm{W}(\cdot)$ denotes the Lambert $\mathrm{W}$-function.

In a recent paper, Nadarajah et al. (2011) used the Lambert W-function to derive the qf of the $\boldsymbol{E} \boldsymbol{L}$ distribution. For any complex $t$, the Lambert $\mathrm{W}$-function is defined as the inverse of the function $g(t)=t \mathrm{e}^{t}$. For more details, see http://mathworld.wolfram.com/LambertW-Function.html. An implementation in R software is available through the LambertW package. See http://cran.rproject.org/web/packages/LambertW/LambertW.pdf.

Using the Lagrange inversion theorem, the power series for the $\mathrm{W}$-function holds:

$$
\mathrm{W}(z)=\sum_{k=1}^{\infty} \frac{(-k)^{k-1}}{k !} z^{k}
$$

By applying (15) in equation (14), we have

$$
Q(u)=-\frac{1}{\alpha}-\frac{1}{\beta}-\frac{1}{\alpha} \sum_{k=1}^{\infty} \frac{(-1)^{k}(-k)^{k-1}}{\beta^{k} k !}(\alpha+\beta)^{k}\left(1-u^{1 / b}\right)^{k / a} \exp [-k(\alpha+\beta) / \beta] .
$$

One of the important applications of equation (14) is to determine the median of the $\mathcal{E} \boldsymbol{g} \mathcal{E} \mathcal{E}$ distribution. The median of $X$, say $M$, is obtained by $M=Q(1 / 2)$.

\subsection{Moments}

The nth moment of $X$ can be derived using the fact that the $\mathcal{E} \boldsymbol{g} \mathcal{E} \mathcal{E}$ density function is a double linear mixture of Erlang densities. Thus, based on (13), the nth moment of $X$ is given by

$$
E\left(X^{n}\right)=\sum_{k=0}^{\infty} \sum_{i=0}^{k+1} v_{k, i} \int_{0}^{\infty} x^{n} \pi(x ; i+1,(k+1) \alpha) \mathrm{d} x .
$$

Further, we have

$$
E\left(X^{n}\right)=\sum_{k=0}^{\infty} \sum_{i=0}^{k+1} \frac{(n+i) ! v_{k, i}}{i ![(k+1) \alpha]^{n}}
$$




\subsection{Moments}

The incomplete moments of a distribution play an important role in applications. The $n$th incomplete moment of $X$ is given by $T_{n}(z)=\int_{0}^{z} x^{n} f(x) \mathrm{d} x$ and using equation (13), we can write

$$
T_{n}(z)=\sum_{k=0}^{\infty} \sum_{i=0}^{k+1} v_{k, i} \int_{0}^{z} x^{n} \pi(x ; i+1,(k+1) \alpha) \mathrm{d} x .
$$

Thus, we can write $\mathrm{T}_{\mathrm{n}}(z)$ as

$$
T_{n}(z)=\sum_{k=0}^{\infty} \sum_{i=0}^{k+1} \frac{\{\Gamma(n+i+1)-\Gamma[n+i+1, z(k+1) \alpha]\} v_{k, i}}{i ![(k+1) \alpha]^{n}}
$$

where $\quad \Gamma(a)=\int_{0}^{\infty} t^{a-1} \mathrm{e}^{-t} \mathrm{~d} t \quad$ is the gamma function and $\quad \Gamma(a, z)=\int_{z}^{\infty} t^{a-1} \mathrm{e}^{-t} \mathrm{~d} t$ denotes the upper incomplete gamma function.

The first incomplete moment of $X$ is important to determine the mean deviations, which can be used to measure the amount of scatter in a population, and the Bonferroni and Lorenz curves, which are useful for applications in areas such as economics, reliability, demography and many others. Setting $n=1$ in equation (16) gives

$$
T_{1}(z)=\sum_{k=0}^{\infty} \sum_{i=0}^{k+1} \frac{\{\Gamma(i+2)-\Gamma[i+2, z(k+1) \alpha]\} v_{k, i}}{i !(k+1) \alpha} .
$$

The mean deviations of $\mathrm{X}$ about the mean $\mu=E(X)$ and about the median $M$ are given by $\delta_{1}=\int_{0}^{\infty}|x-\mu| f(x) \mathrm{d} x$ and $\delta_{2}=\int_{0}^{\infty}|x-M| f(x) \mathrm{d} x$, respectively, where $f(x)$ is the pdf (6). Using equation (17), these measures follow as

$$
\delta_{1}(X)=2\left[\mu F(\mu)-T_{1}(\mu)\right] \quad \text { and } \quad \delta_{2}(X)=\mu-2 T_{1}(M),
$$

where $F(M)$ is the cdf (5) evaluated at $M$ and $T_{l}(z)$ is given by (17).

Equation (17) can also be used to obtain the Bonferroni and Lorenz curves of $X$ given by $B(p)$ $=T_{I}(q) /(p \mu)$ and $L(p)=T_{I}(q) / \mu$, respectively, where $q=Q(p)$ is determined by (14) for a specified probability $p$.

\subsection{Generating function}

The mgf of $\mathrm{X}$ can be determined from (13) as

$$
M(t)=\sum_{k=0}^{\infty} \sum_{i=0}^{k+1} v_{k, i} \int_{0}^{\infty} \mathrm{e}^{t x} \pi(x ; i+1,(k+1) \alpha) \mathrm{d} x .
$$


Then, for all $t<(k+1) \alpha$, we have

$$
M(t)=\sum_{k=0}^{\infty} \sum_{i=0}^{k+1}\left[1-\frac{t}{(k+1) \alpha}\right]^{-(i+1)} v_{k, i} .
$$

\subsection{Rényi entropy}

The entropy of $X$ is a measure of variation of the uncertainty. There are many entropy measures studied and discussed in the literature, but the Rényi entropy is perhaps one of the most popular. The R_enyi entropy of X with density (6) is given by

$$
I_{R}(\rho)=\frac{1}{(1-\rho)} \log \left(\int_{0}^{\infty} f(x)^{\rho} \mathrm{d} x\right)
$$

where $p>0$ and $p \neq 1$.

Now, we consider the generalized binomial expansion

$$
(1-z)^{b}=\sum_{k=0}^{\infty}(-1)^{k}\left(\begin{array}{l}
b \\
k
\end{array}\right) z^{k}
$$

which holds for any real non-integer $b$ and $|z|<1$. Using (19) twice in equation (4), we can write

$$
f(x)^{\rho}=(a b)^{\rho} \sum_{k, \ell=0}^{\infty}(-1)^{k+\ell}\left(\begin{array}{c}
\rho(b-1) \\
k
\end{array}\right)\left(\begin{array}{c}
a k+\rho(a-1) \\
\ell
\end{array}\right) g(x)^{\rho} G(x)^{\ell} .
$$

Inserting (1) and (2) in equation (20) and applying the binomial expansion twice from $\left[\alpha+\beta-(\beta+\bar{\alpha}+\alpha \beta x) \bar{e}^{-\alpha x}\right]^{\ell} \quad$, we obtain

$$
\begin{gathered}
f(x)^{\rho}=(a b)^{\rho} \sum_{k, \ell=0}^{\infty} \sum_{m=0}^{\ell} \sum_{n=0}^{m} \frac{(-1)^{k+\ell+m} \alpha^{2 \rho+n} \beta^{m-n}}{(\alpha+\beta)^{\rho+m}}\left(\begin{array}{c}
\rho(b-1) \\
k
\end{array}\right)\left(\begin{array}{c}
a k+\rho(a-1) \\
\ell
\end{array}\right)\left(\begin{array}{c}
\ell \\
m
\end{array}\right)\left(\begin{array}{c}
m \\
n
\end{array}\right) \\
\times(1+\beta x)^{\rho+n} \mathrm{e}^{-\alpha(\rho+m) x}
\end{gathered}
$$

Further, by inserting (21) in equation (18), the Rényi entropy reduces to 


$$
\begin{aligned}
I_{R}(\rho)=\frac{1}{(1-\rho)} \log \left\{(a b)^{\rho} \sum_{k, \ell=0}^{\infty} \sum_{m=0}^{\ell} \sum_{n=0}^{m} \frac{(-1)^{k+\ell+m} \alpha^{2 \rho+n} \mathrm{e}^{\alpha(\rho+m) / \beta}}{\beta^{n+1-m}(\alpha+\beta)^{\rho+m}}\right. \\
\left.\quad \times\left(\begin{array}{c}
\rho(b-1) \\
k
\end{array}\right)\left(\begin{array}{c}
a k+\rho(a-1) \\
\ell
\end{array}\right)\left(\begin{array}{c}
\ell \\
m
\end{array}\right)\left(\begin{array}{c}
m \\
n
\end{array}\right) \mathbb{E}[-(\rho+n), \alpha(\rho+m) / \beta]\right\},
\end{aligned}
$$

Where $\mathbb{E}[\rho, z]=\int_{1}^{\infty} t^{-\rho} \mathrm{e}^{-z t} \mathrm{~d} t$ is the exponential integral function.

\subsection{Order statistics}

The density function $f_{i: n}(x)$ of the $i$ th order statistic, say $X_{i: n}$, for $i=1, \ldots, n$, from a random sample $X_{l}, \ldots, X_{n}$ having the $\mathcal{E} \boldsymbol{g}$ distribution can be expressed as

$$
f_{i: n}(x)=\frac{f(x)}{B(i, n-i+1)} F(x)^{i-1}[1-F(x)]^{n-i},
$$

where $f(x)$ is the pdf (4) and $F(x)$ is the cdf (3).

Applying the binomial expansion in the last equation, we have

$$
f_{i: n}(x)=\frac{1}{B(i, n-i+1)} \sum_{j=0}^{n-i}(-1)^{j}\left(\begin{array}{c}
n-i \\
j
\end{array}\right) f(x) F(x)^{i+j-1} .
$$

Substituting (3) and (4) in equation (22) and applying the generalized binomial expansion (19), we can write

$$
\begin{gathered}
f_{i: n}(x)=\frac{a b}{B(i, n-i+1)} \sum_{\ell=0}^{\infty} \sum_{j=0}^{n-i} \sum_{k=0}^{\infty}(-1)^{j+k+\ell}\left(\begin{array}{c}
n-i \\
j
\end{array}\right)\left(\begin{array}{c}
b(i+j)-1 \\
k
\end{array}\right)\left(\begin{array}{c}
a(k+1)-1 \\
\ell
\end{array}\right) \\
\times g(x) G(x)^{\ell} .
\end{gathered}
$$

Then, after a simple algebraic manipulation, we have

$$
f_{i: n}(x)=\sum_{\ell=0}^{\infty} q_{\ell} h_{\ell+1}(x),
$$

where $q_{\ell}$ is given by

$$
q_{\ell}=\frac{a b}{B(i, n-i+1)} \sum_{j=0}^{n-i} \sum_{k=0}^{\infty} \frac{(-1)^{j+k+\ell}}{\ell+1}\left(\begin{array}{c}
n-i \\
j
\end{array}\right)\left(\begin{array}{c}
b(i+j)-1 \\
k
\end{array}\right)\left(\begin{array}{c}
a(k+1)-1 \\
\ell
\end{array}\right),
$$

and $h_{\ell+1}(x)$ denotes the exp- $\boldsymbol{g}$ density function with power parameter $\quad \ell+1$ (for $\ell \geq 0$ ). 
Equation (23) reveals that the density function of the $\boldsymbol{\varepsilon} \boldsymbol{g}$ order statistic is a linear mixture of exp- $\boldsymbol{g}$ densities. We emphasize that this result is not new and has already been presented by Cordeiro et al. (2013). However, we now give an alternative way of expressing the weights that compose this linear combination.

By combining equations (11) and (23) and after some algebra, we obtain

$$
f_{i: n}(x)=\sum_{m=0}^{\infty} \sum_{s=0}^{m+1} d_{m, s} \pi(x ; s+1,(m+1) \alpha),
$$

where $\quad d_{m, s}=\sum_{\ell=m}^{\infty} q_{\ell} p_{m, s}^{(\ell+1)}$ the quantity $p_{m, s}^{(\ell+1)}$ is given by (12) and $\pi(x ; s+1 ;(m+1) \alpha)$ denotes the Erlang density with shape parameter $s+1$ and scale parameter $(m+1) \alpha$.

Thus, based on (24), we obtain an important result that gives the density of $X_{i: n}$ as a double linear mixture of Erlang densities. Undoubtedly, there are many applications for equation (24), but the most important is to calculate the moments and the mgf of the ith order statistic. The $r$ th moment of $X_{i: n}$ is given by

$$
E\left(X_{i: n}^{r}\right)=\sum_{m=0}^{\infty} \sum_{s=0}^{m+1} d_{m, s} \int_{0}^{\infty} x^{r} \pi(x ; s+1,(m+1) \alpha) \mathrm{d} x .
$$

Based on the results presented in Section 4.3, the last equation reduces to

$$
E\left(X_{i: n}^{r}\right)=\sum_{m=0}^{\infty} \sum_{s=0}^{m+1} \frac{(r+s) ! d_{m, s}}{s ![(m+1) \alpha]^{r}}
$$

Next, the mgf of $X_{i: n}$ is given by

$$
\varphi(t)=\sum_{m=0}^{\infty} \sum_{s=0}^{m+1} d_{m, s} \int_{0}^{\infty} \mathrm{e}^{t x} \pi(x ; s+1,(m+1) \alpha) \mathrm{d} x .
$$

Based on the results in Section 4.5, the last equation can be rewritten as

$$
\varphi(t)=\sum_{m=0}^{\infty} \sum_{s=0}^{m+1}\left[1-\frac{t}{(m+1) \alpha}\right]^{-(s+1)} d_{m, s},
$$

for all $t<(m+1) \alpha$.

\subsection{Reliability}

Here, we derive the reliability, say $R$, for the $\mathcal{E} \boldsymbol{g} \mathcal{E} \mathcal{E}$ model when $X_{1} \sim \mathcal{E} \boldsymbol{g} \mathcal{E} \mathcal{E}\left(a_{1}, b_{1}, \alpha, \beta\right)$ and $X_{2} \sim \mathcal{E} \boldsymbol{g} \mathcal{E} \mathcal{E}\left(a_{2}, b_{2}, \alpha, \beta\right)$ are two independent random variables with the same baseline parameters $\alpha$ and $\beta$. Let $f_{1}(x)$ denote the pdf of $X_{1}$ and $F_{2}(x)$ denote the cdf of $X_{2}$. The reliability can be expressed as $R=\mathrm{P}\left(X_{1}>X_{2}\right)=\int_{0}^{\infty} f_{1}(x) F_{2}(x) \mathrm{d} x$ and using equations (8) and (9) gives 


$$
R=\sum_{j, k=0}^{\infty} \mathcal{I}_{j, k} \int_{0}^{\infty} h_{j+1}(x) H_{k+1}(x) \mathrm{d} x
$$

where $\mathcal{I}_{j, k}=\sum_{m, n=1}^{\infty}(-1)^{j+k+m+n+2}\left(\begin{array}{c}b_{1} \\ m\end{array}\right)\left(\begin{array}{c}m a_{1} \\ j+1\end{array}\right)\left(\begin{array}{c}b_{2} \\ n\end{array}\right)\left(\begin{array}{c}n a_{2} \\ k+1\end{array}\right)$.

Thus, the reliability of $X$ reduces to

$$
R=\sum_{j, k=0}^{\infty} \sum_{\ell=0}^{j+k+1} \sum_{r=0}^{\ell} \sum_{i=0}^{r+1} \frac{(-1)^{\ell}(j+1) \alpha^{r-i+1} \beta^{\ell+i-r} i !}{(\ell+1)^{i+1}(\alpha+\beta)^{\ell+1}}\left(\begin{array}{c}
j+k+1 \\
\ell
\end{array}\right)\left(\begin{array}{c}
\ell \\
r
\end{array}\right)\left(\begin{array}{c}
r+1 \\
i
\end{array}\right) \mathcal{I}_{j, k} .
$$

\section{Estimation and Inference}

Several approaches for parameter estimation were proposed in the literature but the maximum likelihood method is the most commonly employed. The maximum likelihood estimators (MLEs) enjoy desirable properties and can be used when constructing confidence intervals and regions and also in test statistics. The normal approximation for these estimators in large sample distribution theory is easily handled either analytically or numerically. So, we consider the estimation of the unknown parameters a, b, $\alpha$ and $\beta$ of the $\mathcal{E} \boldsymbol{g} \mathcal{E} \mathcal{E}$ distribution from complete samples only by maximum likelihood. Let $\mathrm{x}_{1}, \ldots, \mathrm{x}_{\mathrm{n}}$ be a random sample of size $\mathrm{n}$ from the $\mathcal{E} \boldsymbol{g} \mathcal{E} \mathcal{E}$ distribution. The $\log$-likelihood function for the vector of parameters $\theta=(\mathrm{a}, \mathrm{b}, \alpha, \beta)^{\mathrm{T}}$, say $\ell(\theta)$ can be expressed as

$$
\begin{aligned}
\ell(\boldsymbol{\theta}) & =n \log \left(a b \alpha^{2}\right)-n a b \log (\alpha+\beta)-a \alpha \sum_{i=1}^{n} x_{i}+(a-1) \sum_{i=1}^{n} \log \left[z\left(x_{i}\right)\right] \\
& +\sum_{i=1}^{n} \log \left(1+\beta x_{i}\right)+(b-1) \sum_{i=1}^{n} \log \left[(\alpha+\beta)^{a}-z\left(x_{i}\right)^{a} \mathrm{e}^{-a \alpha x_{i}}\right],
\end{aligned}
$$

where $z\left(x_{i}\right)=\beta+\alpha+\alpha \beta x_{i}$.

Equation (25) can be maximized either directly by using the Ox program (sub-routine MaxBFGS), R (optim function) and SAS (PROC NLMIXED), or by solving the nonlinear likelihood equations obtained by differentiating . $\ell(\theta)$ The elements of the score vector are given by

$$
\begin{aligned}
\frac{\partial \ell(\boldsymbol{\theta})}{\partial a} & =\frac{n}{a}+(b-1) \sum_{i=1}^{n} \frac{\left\{(\alpha+\beta)^{a} \log (\alpha+\beta)-z\left(x_{i}\right)^{a} \log \left[z\left(x_{i}\right)\right] \mathrm{e}^{-a \alpha x_{i}}+\alpha x_{i} z\left(x_{i}\right)^{a} \mathrm{e}^{-a \alpha x_{i}}\right\}}{\left[(\alpha+\beta)^{a}-z\left(x_{i}\right)^{a} \mathrm{e}^{-a \alpha x_{i}}\right]} \\
& -n b \log (\alpha+\beta)+\sum_{i=1}^{n} \log \left[z\left(x_{i}\right)\right]-\alpha \sum_{i=1}^{n} x_{i}, \\
\frac{\partial \ell(\boldsymbol{\theta})}{\partial b} & =\frac{n}{b}-n a \log (\alpha+\beta)+\sum_{i=1}^{n} \log \left[(\alpha+\beta)^{a}-z\left(x_{i}\right)^{a} \mathrm{e}^{-a \alpha x_{i}}\right],
\end{aligned}
$$




$$
\begin{aligned}
\frac{\partial \ell(\boldsymbol{\theta})}{\partial \alpha} & =\frac{2 n}{\alpha}+(b-1) \sum_{i=1}^{n} \frac{a(\alpha+\beta)^{a-1}-a z\left(x_{i}\right)^{a-1}\left(1+\beta x_{i}\right) \mathrm{e}^{-a \alpha x_{i}}+a x_{i} z\left(x_{i}\right)^{a} \mathrm{e}^{-a \alpha x_{i}}}{\left[(\alpha+\beta)^{a}-z\left(x_{i}\right)^{a} \mathrm{e}^{-a \alpha x_{i}}\right]} \\
& -\frac{n a b}{\alpha+\beta}-a \sum_{i=1}^{n} x_{i}+(a-1) \sum_{i=1}^{n} \frac{\left(1+\beta x_{i}\right)}{z\left(x_{i}\right)}, \\
\frac{\partial \ell(\boldsymbol{\theta})}{\partial \beta} & =-\frac{n a b}{\alpha+\beta}+(b-1) \sum_{i=1}^{n} \frac{a(\alpha+\beta)^{a-1}-a z\left(x_{i}\right)^{a-1}\left(1+\alpha x_{i}\right) \mathrm{e}^{-a \alpha x_{i}}}{\left[(\alpha+\beta)^{a}-z\left(x_{i}\right)^{a} \mathrm{e}^{-a \alpha x_{i}}\right]} \\
& +\sum_{i=1}^{n} \frac{x_{i}}{\left(1+\beta x_{i}\right)}+(a-1) \sum_{i=1}^{n} \frac{\left(1+\alpha x_{i}\right)}{z\left(x_{i}\right)} .
\end{aligned}
$$

The MLE $\hat{\theta}$ of $\theta$ can be obtained numerically. For interval estimation and hypothesis tests on the parameters a, b, $\alpha$ and $\beta$, we determine the $4 \times 4$ observed information matrix given by $\mathrm{J}(\theta)=\left\{-\mathrm{U}_{\mathrm{rs}}\right\}$, whose elements $U_{r s}=\partial^{2} \ell(\boldsymbol{\theta}) /(\partial r \partial s)$ for $r, s \in\{a, b, \alpha, \beta\}$ can be obtained from the authors upon request.

\section{Applications to real data}

Here, we present two applications to real data to illustrate the potentiality of the new distribution. First, in addition to the $\mathcal{E} \boldsymbol{g} \mathcal{E} \mathcal{E}$ model, we consider the three-parameter $\mathcal{E} \boldsymbol{g} \mathcal{E} \mathcal{E}(a, b, \alpha$, $0)$ and $\mathcal{E} \boldsymbol{g} \mathcal{E}(a, b, \alpha, 1)$ sub-models. Also, the three-parameter beta-Lindley $(B L)$ distribution, proposed by Merovci and Sharma (2014), is compared with the $\mathcal{E} \boldsymbol{g} \mathcal{E}$ distribution and its submodels. All computations are performed using the SAS subroutine NLMixed.

The $B L$ density is given by

$$
\eta(x ; a, b, \alpha)=\frac{\alpha^{2}(1+x) \mathrm{e}^{-b \alpha x}}{(\alpha+1)^{a+b-1} B(a, b)}(1+\alpha+\alpha x)^{b-1}\left[\alpha+1-(1+\alpha+\alpha x) \mathrm{e}^{-\alpha x}\right]^{a-1},
$$

where $B(a, b)=\Gamma(a) \Gamma(b) / \Gamma(a+b)$ is the beta function.

First, we consider the number of failures for the air conditioning system of jet airplanes. These data were reported by Cordeiro and Lemonte (2011) and Huang and Oluyede (2014): 194, $413,90,74,55,23,97,50,359,50,130,487,57,102,15,14,10,57,320,261,51,44,9,254$, $493,33,18,209,41,58,60,48,56,87,11,102,12,5,14,14,29,37,186,29,104,7,4,72,270$, $283,7,61,100,61,502,220,120,141,22,603,35,98,54,100,11,181,65,49,12,239,14,18$, $39,3,12,5,32,9,438,43,134,184,20,386,182,71,80,188,230,152,5,36,79,59,33,246$, $1,79,3,27,201,84,27,156,21,16,88,130,14,118,44,15,42,106,46,230,26,59,153,104$, $20,206,5,66,34,29,26,35,5,82,31,118,326,12,54,36,34,18,25,120,31,22,18,216$, $139,67,310,3,46,210,57,76,14,111,97,62,39,30,7,44,11,63,23,22,23,14,18,13,34$, $16,18,130,90,163,208,1,24,70,16,101,52,208,95,62,11,191,14,71$. Some descriptive statistics for these data are given below. The smallest and the largest values are 1 and 603, respectively. Further, the mean, median and variance are 92.07, 54.00 and 11645.93, respectively. 
Table 1 lists the MLEs of the model parameters (with the corresponding standard errors in parentheses) for all fitted models and also the values of the Akaike information criterion (AIC), Bayesian information criterion (BIC) and consistent Akaike information criterion (CAIC) statistics. In general, it is considered that the lower values of these criteria indicates the better fit to the data. The figures in Table 1 revels that the $\mathcal{E} \boldsymbol{g} \mathcal{E} \mathcal{E}$ model has the lowest AIC and BIC values among all fitted models. Thus, the proposed $\mathcal{E} \boldsymbol{g} \mathcal{E} \mathcal{E}$ distribution is the best model to explain these data.

Table 1: The MLEs (and their standard errors in parentheses), AIC, BIC and CAIC statistics for the number of successive failures for the air conditioning system.

\begin{tabular}{lccccccc}
\hline Distribution & $\widehat{a}$ & $\widehat{b}$ & $\widehat{\alpha}$ & $\widehat{\beta}$ & AIC & BIC & CAIC \\
\hline $\mathcal{E G E \mathcal { E }}$ & 0.0639 & 0.6470 & 0.1497 & 183.90 & $\mathbf{2 0 7 7 . 4}$ & $\mathbf{2 0 7 7 . 6}$ & 2090.3 \\
& $(0.0721)^{\mathrm{a}}$ & $(0.1254)$ & $(0.1667)$ & $(1248.4)$ & & & \\
$\mathcal{E} \mathcal{G E}$ & 0.1010 & 0.9100 & 0.1010 & 0 & 2081.5 & 2081.6 & 2091.2 \\
& $(2.3212)^{\mathrm{a}}$ & $(0.0866)$ & $(0.9112)$ & $(-)$ & & & \\
$\mathcal{E} \mathcal{G} \mathcal{L}$ & 0.0565 & 0.6951 & 0.1734 & 1 & 2078.0 & 2078.1 & $\mathbf{2 0 8 7 . 7}$ \\
& $(0.0577)^{\mathrm{a}}$ & $(0.1224)$ & $(0.1758)$ & $(-)$ & & & \\
$\mathcal{B} \mathcal{L}$ & 0.4842 & 0.5379 & 0.0234 & - & 2086.6 & 2086.7 & 2096.3 \\
& $(0.0577)^{\mathrm{a}}$ & $(0.3188)$ & $(0.0132)$ & $(-)$ & & & \\
\hline
\end{tabular}

${ }^{a}$ Denotes the standard deviation of the MLEs.

Further, we consider the formal goodness-of-fit tests based on the Cramér-von Mises $\left(W^{*}\right)$ and Anderson-Darling $\left(A^{*}\right)$ test statistics in order to verify which distribution fits better the current data. The $\mathrm{W} *$ and $A^{*}$ statistics are described in Chen and Balakrishnan (1995). In general, the lower values of these statistics indicate the better fit to the data. Table 2 gives the values of the $W^{*}$ and $A^{*}$ statistics for all fitted models. Based on the figures in this table, we conclude that the $\mathcal{E} \mathfrak{g} \mathcal{E} \mathcal{E}$ distribution provides a better fit to these data than its sub-models and the $B L$ distribution.

Table 2: Goodness-of-fit tests for the number of successive failures for the air conditioning system.

\begin{tabular}{lcc}
\hline \multirow{2}{*}{ Model } & \multicolumn{2}{c}{ Statistics } \\
\cline { 2 - 3 } & $W^{*}$ & $A^{*}$ \\
\hline $\mathcal{E} \mathcal{G} \mathcal{E}$ & $\mathbf{0 . 1 1 3 7}$ & $\mathbf{0 . 7 1 3 6}$ \\
$\mathcal{E} \mathcal{G}$ & 0.1940 & 1.2030 \\
$\mathcal{E} \mathcal{G} \mathcal{L}$ & 0.1415 & 0.8886 \\
$\mathcal{B} \mathcal{L}$ & 0.2491 & 1.5472 \\
\hline
\end{tabular}


Plots of the estimated pdf and cdf of the $\mathcal{E} \boldsymbol{g} \mathcal{E} \mathcal{E}$ distribution and the histogram of the data are displayed in Figure 3. These plots clearly reveal that the $\mathcal{E} \boldsymbol{g} \mathcal{E} \mathcal{E}$ model fits the data adequately and then it can be chosen for modeling these data.

Second, we consider the data presented by Murthy et al. (2004) on the failure times (in weeks) of 50 components. The data are: $0.013,0.065,0.111,0.111,0.163,0.309,0.426,0.535,0.684$, $0.747,0.997,1.284,1.304,1.647,1.829,2.336,2.838,3.269,3.977,3.981,4.520,4.789,4.849$, 5.202, 5.291, 5.349, 5.911, 6.018, 6.427, 6.456, 6.572, 7.023, 7.087, 7.291, 7.787, 8.596, 9.388, 10.261, 10.713, 11.658, 13.006, 13.388, 13.842, 17.152, 17.283, 19.418, 23.471, 24.777, 32.795, 48.105. Some descriptive statistics of these data are presented below. The minimum observed value is 0.013 , while the maximum value is 48.105 . The mean, median and variance are 7.821 , 5.320 and 84.76 , respectively.

For Murthy et al. (2004)'s data, we compare the $\mathcal{E} \boldsymbol{g} \mathcal{E} \mathcal{E}$ model with the $E E$ (Gómez et al., 2014) and Lindley sub-models and other commonly used models in survival analysis, namely the

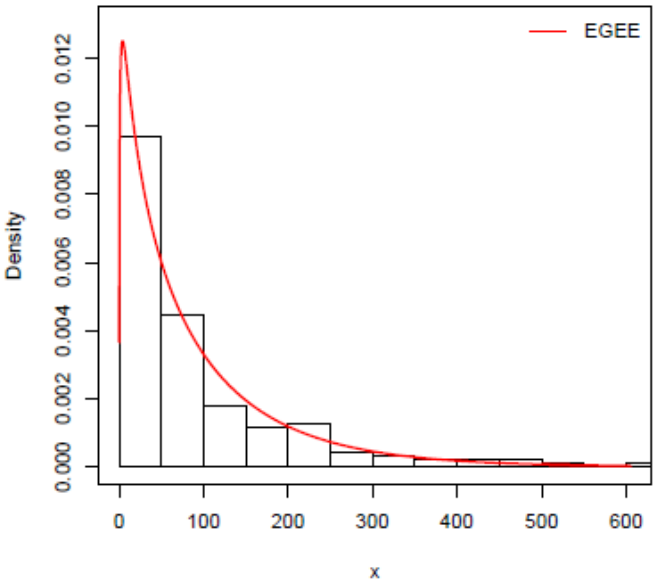

(a) Estimated $\mathcal{E} \mathcal{G} \mathcal{E} \mathcal{E}$ pdf

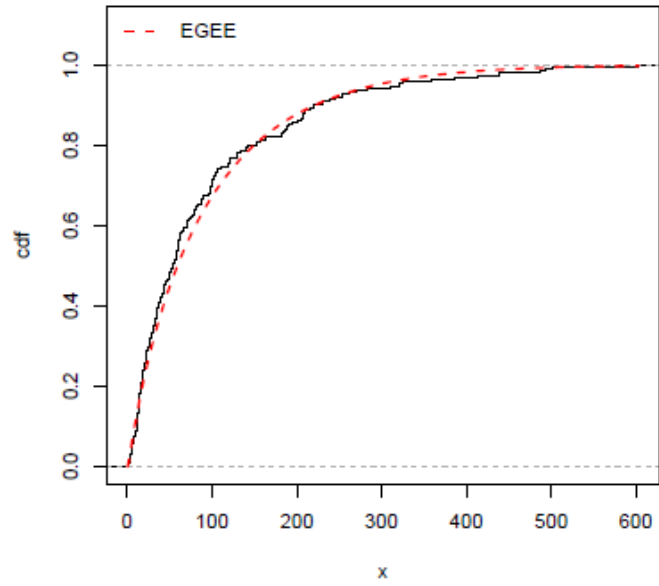

(b) Estimated $\mathcal{E} \mathcal{G} \mathcal{E} \mathcal{E}$ cdf

Figure 3: Plots of the estimated pdf and cdf of the $\mathcal{\varepsilon g} \mathcal{E} \mathcal{E}$ model for the number of successive failures for the air conditioning system.

log-logistic, Fréchet and Birnbaum-Saunders (BS) distributions. The densities of these models are given in the Wolfram alpha website (https://www.wolframalpha.com). Table 3 gives the MLEs of the fitted models to the current data with their corresponding standard errors, in addition to the AIC, BIC and CAIC statistics. Table 4 lists the values of the $A^{*}$ and $W^{*}$ statistics.

Table 3: The MLEs (and their standard errors in parentheses), AIC, BIC and CAIC statistics for the failure times of 50 components. 


\begin{tabular}{|c|c|c|c|c|c|c|c|}
\hline Distribution & $\widehat{\alpha}$ & $\widehat{\beta}$ & $\widehat{a}$ & $\widehat{b}$ & AIC & BIC & CAIC \\
\hline \multirow[t]{2}{*}{$\mathcal{E} \mathcal{G} \mathcal{E} \mathcal{E}$} & 0.3659 & 0.3103 & 0.3239 & 0.6041 & 308.3 & 316.0 & 309.2 \\
\hline & $(0.9972)^{\mathrm{a}}$ & $(0.7977)$ & (1.0123) & $(0.1946)$ & & & \\
\hline \multirow[t]{2}{*}{$\mathcal{E E}$} & 0.1279 & $2.338 \mathrm{E}-7$ & 1 & 1 & 309.7 & 313.5 & 309.9 \\
\hline & $(0.0352)^{\mathrm{a}}$ & $(0.0302)$ & $(-)$ & $(-)$ & & & \\
\hline \multirow[t]{3}{*}{ Lindley } & 0.2317 & 1 & 1 & 1 & 324.6 & 326.5 & 324.6 \\
\hline & $(0.0234)^{\mathrm{a}}$ & $(-)$ & $(-)$ & $(-)$ & & & \\
\hline & $\widehat{\alpha}$ & $\widehat{\beta}$ & & & & & \\
\hline \multirow[t]{3}{*}{ Log-logistic } & 4.0938 & 1.0834 & & & 316.0 & 319.8 & 316.3 \\
\hline & $(0.9218)^{\mathrm{a}}$ & $(0.1304)$ & & & & & \\
\hline & $\widehat{\sigma}$ & $\widehat{\lambda}$ & & & & & \\
\hline \multirow[t]{3}{*}{ Fréchet } & 1.2802 & 0.4791 & & & 341.3 & 345.1 & 341.5 \\
\hline & $(0.4028)^{\mathrm{a}}$ & $(0.0454)$ & & & & & \\
\hline & $\widehat{\alpha}$ & $\widehat{\beta}$ & & & & & \\
\hline \multirow[t]{2}{*}{$\mathcal{B S}$} & 2.7621 & 1.2576 & & & 327.4 & 331.2 & 327.7 \\
\hline & $(0.2973)^{\mathrm{a}}$ & $(0.2721)$ & & & & & \\
\hline
\end{tabular}

a Denotes the standard deviation of the MLE's.

Table 4: Goodness-of-_t tests for the failure times of 50 components.

\begin{tabular}{lcc}
\hline \multirow{2}{*}{ Model } & \multicolumn{2}{c}{ Statistics } \\
\cline { 2 - 3 } & $W^{*}$ & $A^{*}$ \\
\hline $\mathcal{E} \mathcal{G} \mathcal{E} \mathcal{0 . 0 5 1 2}$ & $\mathbf{0 . 2 6 7 0}$ \\
$\mathcal{E \mathcal { E }}$ & 0.0658 & 0.3295 \\
Lindley & 0.0657 & 0.3284 \\
Log-logistic & 0.2572 & 1.3816 \\
Fréchet & 0.6097 & 3.3138 \\
$\mathcal{B S}$ & 0.2794 & 1.5364 \\
\hline
\end{tabular}

The figures in Tables 3 and 4 suggest at least two important conclusions. The first one is that the proposed model $\mathcal{E} g \mathcal{E} \mathcal{E}$ has the lowest values for the AIC, CAIC, $A^{*}$ and $W^{*}$ statistics, and therefore, may be chosen as the best model to analyze the current data. Moreover, these results confirm what has already been demonstrated in the recent statistical literature: generalized models, as the proposed in this paper, usually have superior performance in terms of adjustment when compared to non-generalized models. These conclusions emphasize the importance of the proposed model. 
Finally, Figure 4 displays the estimated pdf and cdf of the $\mathcal{E g} \mathcal{E} \varepsilon$ model and the histogram of the data. These plots reveal that the proposed model is quite suitable for these data.

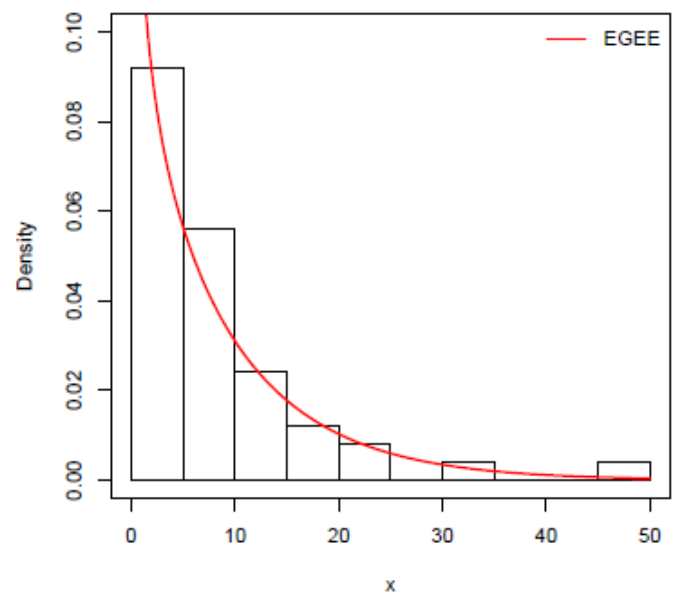

(a) Estimated $\mathcal{E} \mathcal{G} \mathcal{E} \mathcal{E}$ pdf

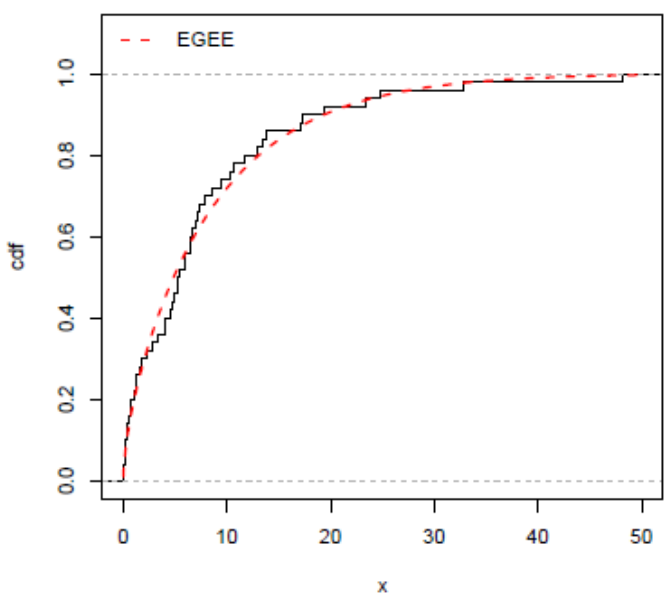

(b) Estimated $\mathcal{E} \mathcal{G} \mathcal{E}$ cdf

Figure 4: Plots of the estimated pdf and cdf of the $\mathcal{E} \boldsymbol{g} \mathcal{E} \mathcal{E}$ model for the failure times of 50 components.

\section{Conclusions}

Recently, Cordeiro et al. (2013) introduced the exponentiated generalized ( $\boldsymbol{\varepsilon g}$ ) class of continuous distributions with two extra shape parameters. In this paper, we consider the $\boldsymbol{\varepsilon} \boldsymbol{g}$ class to generalize the extended exponential $(\mathcal{E} \mathcal{E})$ distribution. We define a new four-parameter lifetime model called the exponentiated generalized extended exponential $(\mathcal{E g} \mathcal{E} \mathcal{E})$ distribution, which includes as special cases the exponential, Lindley and exponentiated exponential distributions, among others. The hazard function of the new model can take the classic bathtub, inverted bathtub, increasing, decreasing and constant shapes. We demonstrate that the $\mathcal{E} \boldsymbol{g} \mathcal{E} \mathcal{E}$ density can be expressed as a double linear mixture of Erlang densities. Further, we derive several basic mathematical properties of the $\mathcal{E} \boldsymbol{g} \mathcal{E} \mathcal{E}$ model, including explicit expressions for the quantile function, ordinary and incomplete moments, mean deviations, Bonferroni and Lorenz curves, generating function, Rényi entropy, density of the order statistics and reliability. We discuss the estimation of the model parameters by maximum likelihood. We conduct two applications to real data to illustrate the flexibility of the new model.

\section{Acknowledgment}

We thank two anonymous referees and the associate editor for their valuable suggestions, which certainly contributed to the improvement of this paper. Additionally, Thiago A. N. de Andrade is grateful the financial support from CAPES (Brazil). 


\section{References}

[1] Chen, G. and Balakrishnan, N. (1995). A general purpose approximate goodness-of-fit test. Journal of Quality Technology, 27:154-161.

[2] Cordeiro, G. M., Gomes, A. E., and da-Silva, C. Q. (2014). Another extended Burr III model: some properties and applications. Journal of Statistical Computation and Simula- tion, $84: 2524-2544$.

[3] Cordeiro, G. M. and Lemonte, A. J. (2011). The $\beta$-Birnbaum-Saunders distribution: An improved distribution for fatigue life modeling. Computational Statistics and Data Analysis, $55: 1445-1461$.

[4] Cordeiro, G. M. and Lemonte, A. J. (2014). The exponentiated generalized BirnbaumSaunders distribution. Applied Mathematics and Computation, 247:762-779.

[5] Cordeiro, G. M., Ortega, E. M. M., and Cunha, D. C. C. (2013). The exponentiated generalized class of distributions. Journal of Data Science, 11:1-27.

[6] da Silva, R. V., Gomes-Silva, F., Ramos, M. W. A., and Cordeiro, G. M. (2015). A new extended gamma generalized model. International Journal of Pure and Applied Mathematics, 100:309-335.

[7] Elbatal, I. and Muhammed, H. Z. (2014). Exponentiated generalized inverse Weibull distribu- tion. Applied Mathematical Sciences, 8:3997-4012.

[8] G'omez, Y. M., Bolfarine, H., and G'omez, H. W. (2014). A new extension of the exponential distribution. Revista Colombiana de Estad'1stica, 37:25-34.

[9] Gupta, R. C., Gupta, P. L., and Gupta, R. D. (1998). Modeling failure time data by Lehmann alternatives. Communications in Statistics-Theory and Methods, 27:887-904.

[10]Gupta, R. D. and Kundu, D. (2001). Exponentiated exponential family: an alternative to gamma and Weibull distributions. Biometrical Journal, 43:117-130.

[11]Huang, S. and Oluyede, B. O. (2014). Exponentiated Kumaraswamy-Dagum distribution with applications to income and lifetime data. Journal of Statistical Distributions and Applications, 1:1-20.

[12]Lehmann, E. L. (1953). The power of rank tests. Annals of Mathematical Statistics, 24:2343.

[13]Lemonte, A. J. (2013). A new exponential-type distribution with constant, decreasing, increas- ing, upside-down bathtub and bathtub-shaped failure rate function. Computational Statistics and Data Analysis, 62:149-170.

[14]Merovci, F. and Sharma, V. K. (2014). The beta-Lindley distribution: properties and applications. Journal of Applied Mathematics, 2014:1-10.

[15]Murthy, D., Xie, M., and Jiang, R. (2004). Weibull models. Wiley series in probability and statistics, John Wiley and Sons, NJ.

[16]Nadarajah, S., Bakouch, H. S., and Tahmasbi, R. (2011). A generalized Lindley distribution. Sankhya B, 73:331-359.

[17]Nadarajah, S. and Haghighi, F. (2011). An extension of the exponential distribution. Statistics: A Journal of Theoretical and Applied Statistics, 45:543-558.

[18] Oguntunde, P. E., Adejumo, A. O., and Balogun, O. S. (2014). Statistical properties of the exponentiated generalized inverted exponential distribution. Applied Mathematics, 4:4755. 
[19]Tahir, H. M. and Nadarajah, S. (2015). Parameter induction in continuous univariate distributions: Well-established G families. Anais da Academia Brasileira de Cincias, 87:539-568.

[20]Zakerzadeh, H. and Dolati, A. (2009). Generalized Lindley distribution. Journal of Mathematical Extension, 3:13-25.

Received April 15, 2015; accepted July 01, 2015.

\author{
Thiago A. N. de Andrade1* \\ Departamento de Estat'istica \\ Universidade Federal de Pernambuco \\ Cidade Universitária - 50740-540, Recife-PE, Brasil. \\ thiagoan.andrade@gmail.com \\ Marcelo Bourguignon $2 \uparrow$ \\ Departamento de Estat'istica \\ Universidade Federal do Rio Grande do Norte \\ Lagoa Nova - 59078-970, Natal-RN, Brasil. \\ m.p.bourguignon@gmail.com \\ Gauss M. Cordeiro3† \\ Departamento de Estat'istica \\ Universidade Federal de Pernambuco \\ Cidade Universitária - 50740-540, Recife-PE, Brasil. \\ gausscordeiro@gmail.com
}


